\title{
CONSÉQUENCES GÉNÉTIQUES DES INTRODUCTIONS D'ESPĖCES DE POISSONS EN L'ABSENCE D'ISOLEMENT REPRODUCTEUR : INTÉRÊT ET LIMITES D'UNE APPROCHE EN MILIEU EXPÉRIMENTAL.
}

\author{
R. GUYOMARD
}

Laboratoire de génétique des poissons, INRA, Jouy-en-Josas, 78352 Cedex, France.

\section{RÉSUMÉ}

Les repeuplements conduisent généralement à la mise en contact de populations conspécifiques génétiquement différenciées et à l'introgression d'un pool génique dans l'autre. Les conséquences de cette introgression sur l'évolution de la variabilité génétique et la biologie de la population en place constituent une question d'intérêt majeur à la fois sur les pians fondamental et appliqué. Cette question peut être abordée en milieu naturel, mais l'étude des performances, en pisciculture, de croisements expérimentaux offre une alternative intéressante. Nous avons appliqué cette approche au cas de la truite commune. Le succès reproducteur et la croissance de croisements expérimentaux de première et deuxième générations entre une souche domestique (origine atlantique) et une souche sauvage méditerranéenne de truite commune (distance génétique standard de $\mathrm{Nei}=0.10$ ) ont été comparés. Les résultats obtenus ne mettent pas en évidence d'incompatibilité génomique entre les deux formes et nous conduisent à penser que les phénomènes sélectifs susceptibles d'agir en milieu naturel, lors d'une introgression entre ces deux formes, ne peuvent être que de faible intensité. Les limites de cette approche pour évaluer les conséquences génétiques des repeuplements et son intérêt pour la gestion des populations sont discutés.

\section{CONSEQUENCES OF FISH INTRODUCTION IN THE ABSENCE OF REPRODUCTIVE ISOLATION : INTEREST AND LIMITS OF THE EXPERIMENTAL APPROACH.}

\begin{abstract}
Stocking generally results in artificial secondary contact and introgression between differentiated conspecific populations. The consequence of such introgressions on the genetic and phenotypic variation in wild stocks is an important concern of population genetics and fisheries management. Introgression can be studied directly on natural populations, but the comparison of the fitness of artificial crosses under experimental conditions offers an interesting alternative approach. In this paper, we report data on growth performance and reproductive success of first and second generation crosses between a domesticated stock and a wild Mediterranean population of brown trout (Nei's standard genetic distance between the two taxa $=0.10$ ). Our results did not reveal any genomic incompatibility between the two taxa. They support the idea that selective effects are weak or non-existent in introgressed Mediterranean populations. In such conditions, low and temporary gene flow between distinct geographic entities could be beneficial to natural populations, and might be artificially established provided that it is compatible with the conservation of the genetic resources of the species. These recommendations hold for other species under similar circumstances.
\end{abstract}




\section{INTRODUCTION}

Lorsque l'introduction conduit à mettre en contact deux populations appartenant à la même espèce, il faut s'attendre à des phénomènes d'hybridation, puis d'introgression, qui peuvent aboutir à un brassage complet des gènes des deux populations. Ce type de situation pose deux problèmes principaux.

Le premier est celui de la mise en évidence de l'hybridation et de l'introgression et de l'estimation du taux de "contamination " génétique. Ce problème est d'autant plus facile à résoudre que les deux populations mises en présence sont génétiquement différenciées. Dans le cas de la truite commune, il est facile de mettre en évidence l'introgression entre souches domestiques d'origine atlantique et populations méditerranéennes, grâce à deux locus protéiques fixés (ou presque) pour des allèles différents dans les deux types de populations (BARBATLETERRIER et al., 1989 ; GUYOMARD, 1989 ; BEAUDOU et al., 1993 ; PRESA et al., 1994). Par contre, la faible différenciation généralement observée entre populations domestiques et naturelles atlantiques ne permet pas de conclure sur le degré de contamination de ces dernières. L'efficacité de nouvelles méthodes de marquage génétique pour l'estimation du taux de contamination génétique par le repeuplement dans la zone atlantique est en cours d'évaluation.

Le second problème est celui des conséquences de l'introgression sur la biologie et la valeur sélective de la population en place. Cette question est beaucoup plus difficile à traiter. Elle peut être abordée par deux approches différentes. La première est l'étude de situations " historiques" de repeuplement mises en évidence dans le milieu naturel. Ces situations offrent un accès immédiat à l'étude in situ des effets après plusieurs générations de repeuplement. Lorsque de nombreuses situations historiques ont été identifiées, il est toujours possible de réaliser une sélection de situations à étudier en fonction de divers critères. Par contre, l'interprétation des résultats d'analyses statistiques réalisées sur des échantillons naturels est très difficile en raison de la complexité spatiale et temporelle de la structure génétique des populations dont ils sont issus, du nombre de facteurs impliqués dans l'évolution des populations et de l'absence d'une description avant repeuplement. II est donc, en général, impossible de trancher entre des hypothèses de type neutraliste (par exemple, le repeuplement n'a pas d'effet sur la valeur sélective des populations naturelles) et sélectionniste (existence d'effets sélectifs). La seconde approche est l'expérimentation, qui peut être réalisée en conditions naturelles, semi-naturelles (par exemple, chenal de fraie) ou en pisciculture. L'expérimentation permet de contrôler un certain nombre de facteurs (choix des stades et lieux de repeuplement, identification génétique préalable et choix des souches naturelles et domestiques, etc.) et de suivre la phase initiale de repeuplement. Ici, nous ne considérons qu'un exemple très simple d'analyse de performances de croissance et de succès reproducteur de croisements entre une souche domestique et une population méditerranéenne, c'est-à-dire deux taxons présentant déjà un niveau de différentiation relativement élevé pour deux populations conspécifiques (distance génétique standard de Nei entre les deux taxons $=0.10$, GUYOMARD, 1989). Nous discutons ensuite brièvement de l'intérêt et des limites de l'expérimentation pour l'étude des conséquences génétiques de l'introgression.

\section{MATÉRIEL ET MÉTHODES}

Un croisement diallèle entre une souche domestique et une souche méditerranéenne a été réalisé (tableau I). Les géniteurs domestiques proviennent d'une souche domestique entretenue à la pisciculture expérimentale de I'INRA et présentant la variabilité électrophorétique classiquement observée dans les populations domestiques de truite commune (PRESA et al., 1994 ; GIUFFRA et al., 1996). Les géniteurs méditerranéens ont été prélevés sur la Reverotte (bassin du Doubs). Leur appartenance à la sous-espèce méditerranéenne a été vérifiée à l'aide de différents marqueurs allozymiques (deux locus : $L D H-5^{*}$ et $T F^{*}$ ) et microsatellites (5 locus) qui n'ont révélé aucune contamination génétique des repeuplements. Les huit lots produits (quatre types de croisements répliqués) ont été incubés et élevés à la pisciculture expérimentale de I'INRA. Les taux de survie des différents lots ont été suivis jusqu'à 126 jours après la fécondation 
(90 jours après l'éclosion). Au delà, seul le lot femelle domestique $x$ mâle Doubs (DS) a été conservé. Les back-crosses ont été réalisés entre mâles DS et femelles domestiques (backcross FDxMH) et femelles DS et mâles domestiques (back-cross FHxMD) et incubés à $10^{\circ} \mathrm{C}$ constants. Les taux de survie ont été mesurés au stade mi-resorption (56 jours après la fécondation). Les taux de survie entre lots ou back-crosses individuels ont été comparés par test de chi-deux ou par analyse de variance à deux facteurs avec réplicats.

\section{Tableau I}

Plan de croisement pour la production des F1. FD = femelles domestiques; FS = femelles sauvages $; M D=$ mâles domestiques $;$ MS = mâles sauvages. No = nombre initial d'oeufs. Entre parenthèses, pourcentage d'embryons vivants au stade oeillé.

Table I

Experimental design for the production of F1. FD = domesticated females ; FS = wild females ; MD = domesticated males $; M S=$ wild males. No = initial number of eggs. In brackets, percentage of surviving embryos at eyed stage.

\begin{tabular}{lcc}
$\begin{array}{l}\text { Type et nombre } \\
\text { de mâles }\end{array}$ & \multicolumn{2}{c}{ Type et nombre de femelles } \\
\hline & FD $(n=5)$ & FS $(n=8)$ \\
FD $(n=10)$ & No $=1008$ & FSxMD \\
& $(80.3 \%)$ & No $=1663$ \\
& FDxMS & $(87.4 \%)$ \\
MS $(n=11)$ & No $=1019$ & FSxMS \\
& $(80.5 \%)$ & No $=2397$ \\
\end{tabular}

\section{RÉSULTATS}

\section{Survie des F1 au stade oeillé}

Les taux de survie et les nombres initiaux d'oeufs fécondés sont présentés dans le tableau I. Les tests de chi-deux (1 ddl) sur les taux de survie ne sont pas significatifs entre lots issus du même type de femelles ( $p=0.17$ pour SD et $S S ; p=0.91$ pour DD et $D S$ ), mais sont hautement significatifs entre lots issus du même type de mâles $(p<0.001)$. Ces différences significatives sont attribuables à un effet maternel non génétique (effet de la qualité de l'oeuf).

\section{Survie et croissance des F1 de l'éclosion à 126 jours}

L'évolution de la survie sur cette période est illustrée par la figure 1. L'analyse de variance à deux facteurs ne fait apparaître aucun terme (mâle, femelle ou interaction) significatif, à l'exception de l'interaction mâle $x$ femelle qui est significative à 126 jours $(p<0.03)$. Cette interaction peut résulter des taux de survie plus élevés des deux types d'hybrides par rapport aux deux lots parentaux. La croissance des F1 est présentée sur la figure 2. Les lots domestiques montrent une croissance supérieure à celle des hybrides, qui ont eux-mêmes une croissance supérieure à celle des lots sauvages. L'analyse de variance à deux facteurs du poids à 126 jours fait apparaître un effet mâle significatif $(p<0.01)$ et un effet femelle non significatif $(p=0.07)$. L'absence d'effet significatif femelle pour la croissance pourrait s'expliquer par le fait que les effets maternels non génétiques, favorables aux descendants de femelles sauvages, pourraient contrebalancer les effets génétiques, favorables aux descendants de femelles domestiques, au cours de la période considérée. 


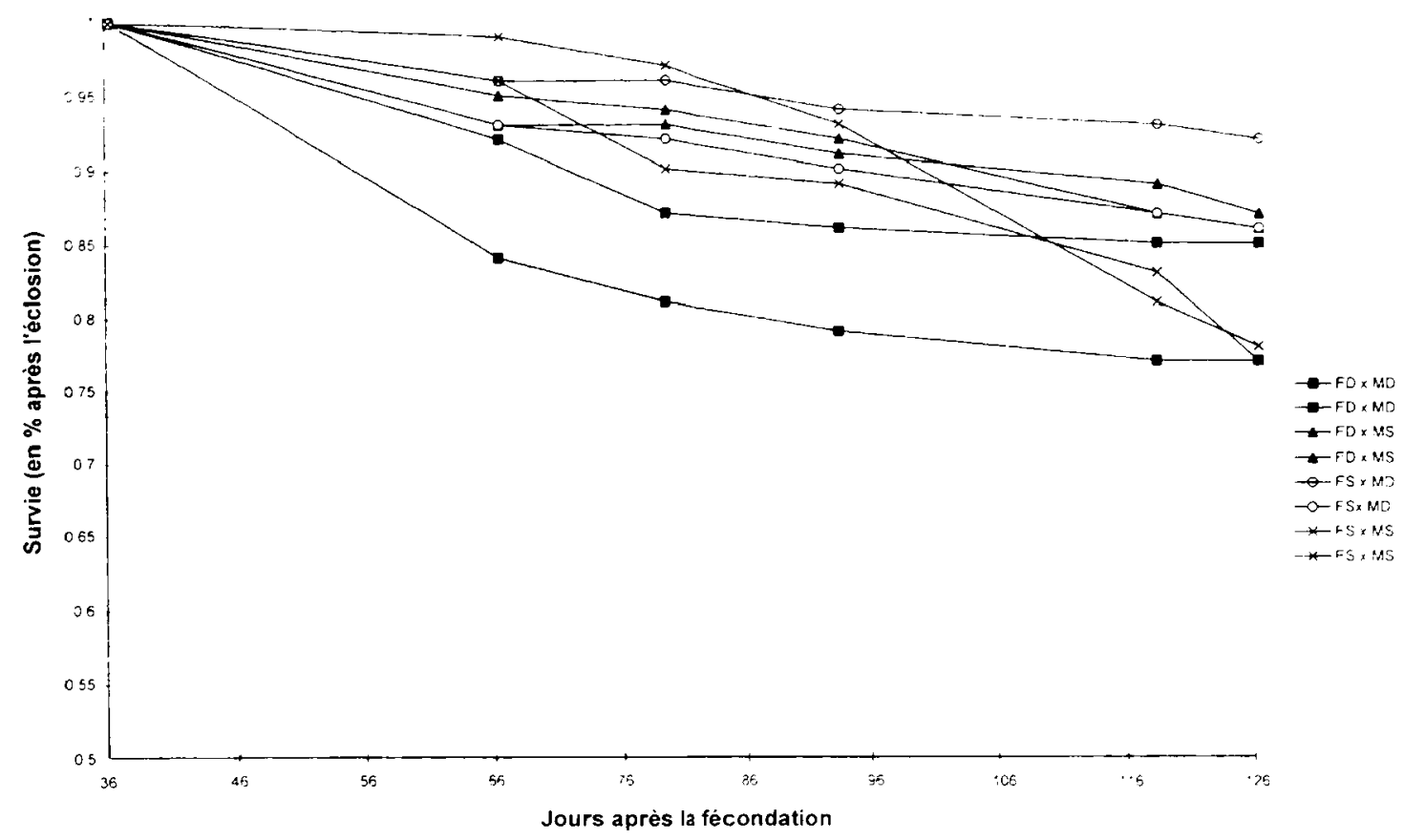

Figure 1

Taux de survie dans les quatre lots F1 en fonction du nombre de jours après la fécondation. L'identification des géniteurs est identique à celle du tableau $\mathbf{I}$.

Figure 1

Evolution of the survival rate in the F1 during the 126 days following the fertilization date. Parents are identified as described in table $\mathrm{I}$.

\section{Survie des back-crosses}

Le tableau II donne la survie des back-crosses ainsi que des témoins domestiques purs (MD x FD) au stade oeillé et à mi-résorption. Les taux de survie sont excellents dans la majorité des cas. II y a une variation notable des taux de survie à 56 jours entre back-crosses issus de femelles domestiques. Ces variations semblent pouvoir être à nouveau attribuables à des effets maternels non génétiques, car ces taux de survie ne sont pas significativement différents de ceux des témoins issus des mêmes femelles à l'exception du lot MH5xFD4. Les back-crosses issus de femelles hybrides présentent tous des taux de survie élevés (> $86 \%$ ).

\section{DISCUSSION}

Les taux de survie très élevés de F1 et back-crosses, présentés ci-dessus, signifient qu'il n'y a pas, ou pratiquement pas, de mortalité sélective des génotypes et nous permettent de considérer les incompatibilités génétiques entre le génome de la souche domestique et celui de la population méditerranéenne, si elles existent, comme négligeables. Nous avons même observé une supériorité statistiquement significative des hybrides F1 par rapport aux deux lots parentaux pour le taux de survie. Des résultats analogues ont été obtenus sur des croisements expérimentaux impliquant des taxons présentant parfois des niveaux de différenciation bien supérieurs à ceux qui sont observés entre souches domestiques et populations méditerranéennes de truite commune (CHEVASSUS, 1979; HALLIBURTON et al., 1983; FERGUSON et al., 1985b et c; ALLENDORF et LEARY, 1986; FERGUSON et al., 1988; GUYOMARD et FORNERIS, résultats non publiés). Bien qu'en théorie il suffise d'une différence à un seul locus pour engendrer une incompatibilité génétique entre deux génomes, les données expérimentales semblent montrer qu'incompatibilités génétiques et anomalies de développement ne se manifestent pas de façon notable tant que les deux génomes n'ont pas 


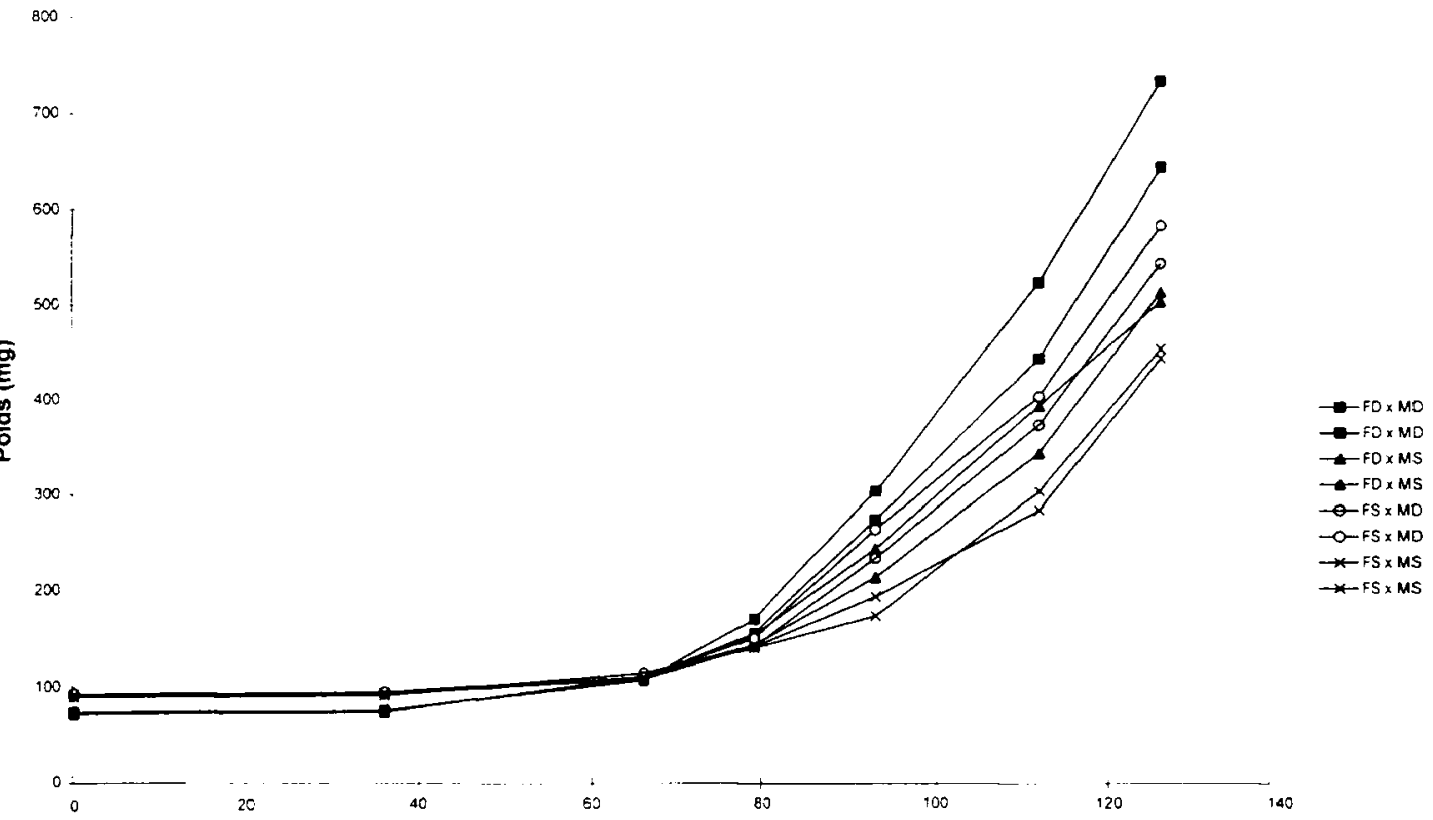

Figure 2

Jours après la fécondation

Evolution du poids individuel moyen en fonction du nombre de jours après la fécondation. L'identification des géniteurs est identique à celle du tableau I.

\section{Figure 2}

Evolution of growth in the F1 groups during the 126 days following fertilization. Parents are identified as described in table $I$.

divergé de façon très importante. Ces résultats nous conduisent à privilégier l'hypothèse selon laquelle les phénomènes sélectifs dus à la rupture d'ensemble de gènes coadaptés consécutifs à l'hybridation, en milieu naturel, entre souches domestiques et méditerranéennes ne peuvent être que de faible intensité. Pour mesurer de façon plus précise le degré d'incompatibilité génétique entre génomes et détecter d'éventuels effets sélectifs faibles liès à cette incompatibilité, des études expérimentales sur la cinétique du développement embryonnaire, l'ontogenèse de la régulation des gènes, le degré d'asymétrie morphologique ou les relations entre niveau d'hétérozygotie et performances pour différentes composantes de la valeur sélective ont été entreprises chez les salmonidés. Les résultats obtenus à partir de ce type d'études se sont avérés différents selon le type de croisement et le critère considérés et n'ont pas permis de dégager de règle générale (HALLIBURTON et al., 1983 ; FERGUSON et al., 1985 $a$, b et c ; LEARY et al., 1985 ; FERGUSON et al., 1988). De plus, elles ont été strictement limitées à l'étude de croisements $F 1$. Seule l'existence d'une relation significative entre niveau d'hétérozygotie et performances pour diverses composantes de la valeur sélective a été très fréquemment mise en évidence (ALLENDORF et LEARY, 1986). Ce type d'approche mériterait d'être développé dans le cas de la truite commune.

Si l'expérimentation en pisciculture nous semble particulièrement adaptée à l'étude des phénomènes d'incompatibilité génétique entre génomes et de stabilité de développement des hybrides, il est cependant clair que cette approche présente certaines limites. Elle ne permet pas de mettre en évidence la mise en place éventuelle de barrières reproductives de type comportemental (appariement préférentiel des individus de même génotype). D'autre part, les coefficients de sélection peuvent varier en fonction de l'environnement et il est donc nécessaire d'évaluer aussi le rôle et les modalités de la sélection directement en milieu naturel. La mise en évidence de phénomènes sélectifs en milieu naturel constitue un objectif d'un intérêt considérable sur le plan académique, mais difficile à atteindre pour deux raisons majeures. D'une part, ainsi que nous l'avons déjà mentionné, il est difficile de faire la part entre les effets 
Tableau II

Taux de survie au stade oeillé et à 56 jours après la fécondation de deux types de back-crosses et leurs témoins domestiques (cf texte pour l'identification des différents lots).

Table II

Survival rates at eyed stage and 56 days after fertilization in two types of back-crosses and their domesticated controls (cf text for identification code of the different batches).

\begin{tabular}{lcc}
\hline GROUPES & $\begin{array}{c}\text { Taux de survie (\%) } \\
\text { stade oeillé }\end{array}$ & $\begin{array}{c}\text { Taux de survie (\%) } \\
56 \text { jours }\end{array}$ \\
\hline MD1xFD1 & 84.3 & 68.2 \\
MD2xFD3 & 90.8 & 80.8 \\
MD3xFD4 & 93.0 & 84.5 \\
MH1xFD1 & 86.6 & 77.9 \\
MH2xFD3 & 91.9 & 84.5 \\
MH3xFD4 & 93.5 & 84.4 \\
MD1xFH1 & 95.0 & 87.4 \\
MD2xFH2 & 92.9 & 88.3 \\
MD3xFH3 & 97.7 & 94.4 \\
MD4xFD4 & 90.3 & 82.5 \\
MD5xFD5 & 63.3 & 56.7 \\
MD4xFH4 & 95.2 & 92.4 \\
MD4xFH5 & 86.4 & 86.4 \\
MD5xFH6 & 95.7 & 94.7 \\
MD5xFH7 & 95.5 & 94.5 \\
MD5xFH8 & 97.1 & 95.3 \\
MH4xFD4 & 88.6 & 81.7 \\
MH5xFD4 & 95.3 & 66.7 \\
MH6xFD5 & 63.7 & 60.6 \\
MH7xFD5 & 66.2 & 64.6 \\
MH8xFD5 & 61.6 & 58.5 \\
\hline & &
\end{tabular}

de la sélection et d'autres facteurs d'évolution comme la dérive génétique et la migration dans le traitement des données populationnelles. D'autre part, en l'absence de phénomènes d'incompatibilité génomique notable dans les croisements expérimentaux, il est probable que les forces sélectives impliquées lors de l'introgression entre souches domestiques et méditerranéennes soient de faible intensité et donc difficiles à détecter (et le soient de plus en plus au fil des générations).

Sur le plan de la gestion génétique des populations, les conséquences du repeuplement peuvent être considérées selon deux critères. Si le critère de gestion de la variabilité est la propagation des souches les plus performantes, il n'est pas exclu que les tests en milieu naturel conduisent à retenir des souches domestiques. Même si le brassage des deux pools géniques engendre des combinaisons de gènes domestiques-sauvages défavorables et une dépression de performances de certaines composantes de la valeur sélective à court terme, il est aussi probable qu'il fait émerger des associations susceptibles d'accroître la valeur sélective et la capacité d'adaptation à moyen ou long terme des stocks naturels méditerranéens. La présence d'une certaine proportion (variable selon les locus) de gènes domestiques ou atlantiques dans les stocks sauvages méditerranéens pourrait être bénéfique pour ces stocks. Selon le critère de conservation patrimoniale à long terme, le repeuplement se traduit par une perte de variabilité interpopulation et, à terme, peut aboutir à la disparition de gènes et génotypes originaux. 
Actuellement, la grande majorité des populations méditerranéennes présente des taux de contamination par les souches domestiques plus ou moins importants et l'accent devrait plutôt être mis sur le second critère, celui de la gestion patrimoniale et de la protection d'un certain nombre de populations non contaminées.

Les stratégies de gestion des ressources génétiques des espèces doivent être élaborées au cas par cas pour tenir compte de leur structure génétique et de leur biologie propres. Cependant, un certain nombre de considérations formulées dans le cas de la truite commune sont généralisables. La structuration de la variabilité génétique résulte toujours de la mutation, de la recombinaison, de la migration, des fluctuations aléatoires des fréquences de gènes et de la sélection et ne correspond pas nécessairement à une distribution optimale des génotypes sur le plan adaptatif. Il peut donc être avisé de tenter de corriger les conséquences du hasard en permettant une diffusion limitée des génotypes. Cette diffusion ne devrait cependant être entreprise que si l'on dispose de moyens permettant, parallèlement, de garantir une bonne conservation du patrimoine génétique de l'espèce.

\section{REMERCIEMENTS}

L'auteur remercie tout particulièrement le président et les gardes-pêche de la fédération départementale de pêche et de pisciculture du Doubs, pour l'intérêt qu'ils ont porté à ce travail ainsi que pour l'aide à l'échantillonnage.

\section{BIBLIOGRAPHIE}

ALLENDORF F.W., LEARY R.F., 1986. Heterozygoty and fitness in populations of animals. In Conservation biology. The science of scarcity and diversity. SOULÉ M.E. ed., Sinauer Associates, Inc., sunderland, MA, 57-76.

BARBAT-LETERRIER A., GUYOMARD R., KRIEG F., 1989. Introgression between introduced domesticated strains and Mediterranean native populations of brown trout (Salmo trutta L.). Aquat. Living Resour., 2, 215-223.

BEAUDOU D., CATTANEO-BERREBI G., POTEAUX C., BERREBI P., 1993. Impact génétique de la réintroduction de truite commune (Salmo trutta fario) dans des populations du sud de la France. Bull. Soc. Zool. Fr., Evol. Zool., 118, 111-112.

CHEVASSUS B., 1979. Hybridization in salmonids : results and perspectives. Aquaculture, 17, 113-128.

FERGUSON M.M., DANZMANN R.G., ALLENDORF F.W., 1985 a. Developmental divergences among hatchery strains of rainbow trout (Salmo gairdneri). I. Pure strains. Can. J. Genet. Cytol., 27, 298-307.

FERGUSON M.M., DANZMANN R.G., ALLENDORF F.W., 1985 b. Developmental divergences among hatchery strains of rainbow trout (Salmo gairdneri). II. Hybrids. Can. J. Genet. Cytol., 27, 298-307.

FERGUSON M.M., DANZMANN R.G., ALLENDORF F.W., 1985 c. Absence of developmental incompatibility in hybrids between rainbow trout and two species of cutthroat trout. Biochem. Genet., 23, 557-570.

FERGUSON M.M., DANZMANN R.G., ALLENDORF F.W., 1988. Developmental success of hybrids between two taxa of salmonids with moderate structural gene divergence. Can. J. Zool., 66, 1389-1395.

GIUFFRA E., GUYOMARD R., FORNERIS G., 1996. Phylogenetic relationships and introgression patterns between incipient parapatric species of Italian brown trout (Salmo trutta L. complex). Molecular ecology, 5, 207-220. 
GUYOMARD, 1989. Diversité génétique de la truite commune. Bull. Fr. Pêche Piscic., 314, 118135.

HALLIBURTON R., PIPKIN R.E., GALL G.A.E., 1983. Reproductive success of artificially hybridized golden trout (Salmo aguabonita) and rainbow trout (Salmo gairdneri). Can. J. Fish. Aquat. Sci., 40, 1264-1269.

LEARY R.F., ALLENDORF F.W., KNUDSEN K.L., 1985. Developmental instability and high meristic counts in interspecific hybrids of salmonid fishes. Evolution, 39, 1318-1326.

PRESA P., KRIEG F., ESTOUP A., GUYOMARD R., 1994. Diversité et gestion génétique de la truite commune : apport de l'étude du polymorphisme des locus protéiques et microsatellites. Genet. Sel. Evol., 26 (Suppl. 1), 183-202. 